\title{
Clinical significance of radiation-induced CD133 expression in residual rectal cancer cells after chemoradiotherapy
}

\author{
AYA KAWAMOTO ${ }^{1}$, KOJI TANAKA ${ }^{1}$, SUSUMU SAIGUSA ${ }^{1}$, YUJI TOIYAMA ${ }^{1}$, YUHKI MORIMOTO ${ }^{1}$, \\ HIROYUKI FUJIKAWA ${ }^{1}$, TAKASHI IWATA ${ }^{1}$, KOHEI MATSUSHITA ${ }^{1}$, TAKESHI YOKOE ${ }^{1}$, \\ HIROMI YASUDA ${ }^{1}$, YASUHIRO INOUE ${ }^{1}$, CHIKAO MIKI $^{1}$ and MASATO KUSUNOKI ${ }^{1,2}$ \\ Departments of ${ }^{1}$ Gastrointestinal and Pediatric Surgery, and ${ }^{2}$ Innovative Surgery, Division of Reparative Medicine, \\ Institute of Life Sciences, Mie University Graduate School of Medicine, Mie 514-8507, Japan
}

Received September 30, 2011; Accepted December 22, 2011

DOI: $10.3892 /$ etm.2011.438

\begin{abstract}
CD133 and CD44 have been considered as markers for colorectal cancer stem cells (CSCs). The association of CD133 and CD44 expression with radiation has not been fully examined in rectal cancer. Both CD133 (PROM) and CD44 mRNA levels were measured in post-chemoradiotherapy (CRT) specimens of 52 rectal cancer patients using real-time RT-PCR and compared to clinicopathological variables and clinical outcome. Their protein levels were examined in the radiationtreated HT29 human colon cancer cell line. Post-CRT CD133 in residual cancer cells was significantly higher than matched pre-CRT CD133 in biopsy specimens $(n=30)$. By contrast, CD44 was significantly lower in post-CRT specimens $(\mathrm{P}<0.01)$. CD133 was associated with distant recurrence after CRT followed by surgery $(\mathrm{P}<0.05)$. Patients with elevated CD133 in residual cancer cells showed poor disease-free survival $(\mathrm{P}<0.05)$. No significant association between post-CRT CD44 and clinical outcome was found. The in vitro study showed that CD133 protein was increased in a radiation dose-dependent manner, despite of the decreased number of clonogenic radiationsurviving cells. CD44 protein was decreased after irradiation. CD133, but not CD44, was increased in radiation-resistant surviving colon cancer cells. Post-CRT CD133 in residual cancer cells may predict metachronous distant recurrence and poor survival of rectal cancer patients after CRT.
\end{abstract}

\section{Introduction}

Recent research has demonstrated that cancer stem cells (CSCs) appear to be particularly resistant to conventional chemo- and

Correspondence to: Dr Aya Kawamoto, Department of Gastrointestinal and Pediatric Surgery, Division of Reparative Medicine, Institute of Life Sciences, Mie University Graduate School of Medicine, 2-174 Edobashi, Tsu, Mie 514-8507, Japan E-mail: ayayaka@clin.medic.mie-u.ac.jp

Abbreviations: RT-PCR, reverse transcription-polymerase chain reaction

Key words: CD133, CD44, chemoradiotherapy, rectal cancer radiation therapies compared to the non-CSC compartment. Conventional cytotoxic therapies initially shrink the bulk of a tumor, but fail to eradicate it, resulting inevitably in tumor recurrence. Treatment failure may in part be due to preferential resistance of CSCs to chemotherapeutic agents $(1,2)$. Bao et al demonstrated that the population of cells enriched for glioma CSCs was dramatically increased by irradiation, and that radioresistant gliomas showed an increased percentage of CD133-positive cells (3). Tsuchida et al showed that anticancer drug treatment increases the side-population fraction (considered as CSCs) in cancer cell lines (4). Accordingly, we also hypothesized that chemoradiotherapy (CRT) decreased non-CSCs which are sensitive to CRT, while it increased the percentage of putative CSCs characteristic of CRT resistance in the population of residual cancer cells.

A cell surface protein, CD133, known as prominim-1, is regarded as one of the most important markers of colon CSCs as reported by two studies which demonstrated that CD133expressing cells sorted by a fluorescence-activated cell sorting system exhibited tumor-forming abilities in xenografts $(5,6)$. A transmembrane glycoprotein molecule, CD44, is a widely expressed cell surface hyaluronan receptor found in normal epithelial, mesenchymal and hematopoietic cells. CD44 has also been reported as an important cell surface marker for isolating colon CSCs (7-9). Previous reports have demonstrated that both CD133 and CD44 are of functional importance for colon CSCs. However, it remains to be resolved whether CD133 or CD44 expression is clinically important in CRT-surviving cells (residual cancer cells after CRT), or whether CRT may increase the expression of these markers.

In this study, we investigated both CD133 and CD44 expression in residual cancer cells after CRT, and evaluated their association with clinicopathological variables and clinical outcome of rectal cancer patients. We also examined whether irradiation by itself induces these markers in a human colon cancer cell line.

\section{Materials and methods}

Patients. A total of 52 patients with advanced rectal cancer were included. All patients were treated with pre-operative CRT followed by surgery at the Department of Gastrointestinal 
and Pediatric Surgery, Mie University Graduate School of Medicine. Formalin-fixed paraffin-embedded (FFPE) specimens were available for this study. Thirty endoscopic tumor biopsy specimens before CRT matched to the FFPE specimens were snap frozen in liquid nitrogen and kept at $-80^{\circ} \mathrm{C}$ until use. All patients signed informed consent forms for their tissue to be used in this study. Selection criteria were the availability of isolated RNA with quality suitable for real-time PCR and complete clinical data.

Treatment. Pre-operative CRT regimen included 4 cycles of 5 -fluorouracil (5FU) administered as $600 \mathrm{mg} / \mathrm{m}^{2}$ for $24 \mathrm{~h}$ on a continuous intravenous route, and tegafur-uracil (UFT) administered as $400 \mathrm{mg} / \mathrm{m}^{2}$ for 5 days orally, with concurrent 20-45 Gy pelvic radiotherapy using a four-field box technique. All patients underwent computed tomography simulation for three-dimensional radiotherapy planning and were treated with a 10-MV photon using a linear accelerator. The radiation field encompassed a volume that included the primary tumor, mesorectum, presacral space, whole of the sacral hollow and regional lymph nodes. The superior border was placed at L5/S1 and the inferior border at $\geq 3 \mathrm{~cm}$ caudal to the primary tumor. The regimen of chemotherapy was based on the combination of continuous infusion of 5FU and UFT (10). The time interval between pre-operative CRT and rectal resection with total mesorectal excision (TME) was 2-6 weeks. No delay of each treatment due to severe toxicity was experienced. All patients underwent standard surgery, including TME, and received 5FU-based adjuvant chemotherapy after surgery for 6 months to 1 year.

Pathological evaluation and treatment response. Pathological evaluation in the resection specimens was performed according to TNM classification (11). Tumor regression of the primary tumor was semi-quantitatively determined by the amount of viable tumor vs. the amount of fibrosis. This ranged from no evidence of any treatment effect to a complete response with no viable tumor identified, as described by Dworak et al (12). Tumor regression grade (TRG) 0 was defined as no regression; TRG1, minor regression (dominant tumor with fibrosis in $\leq 25 \%$ of the tumor mass); TRG2, moderate regression (dominant tumor with fibrosis in $26-50 \%$ of the tumor mass); TRG3, good regression (>50\% tumor regression); and TRG4, total regression (no viable tumor cells, only fibrotic mass).

Microdissection in FFPE specimens. Tumor specimens were fixed in $10 \%$ formaldehyde solution and embedded in paraffin. Sections of FFPE specimens $(10-\mu \mathrm{m})$ were stained with nuclear fast red and subsequently manually microdissected to collect residual cancer and stromal cells, with reference to H\&E sections.

RNA extraction from FFPE specimens. Microdissected samples were digested with proteinase $\mathrm{K}$ in lysis buffer containing Tris- $\mathrm{HCl}$, EDTA and sodium dodecyl sulfate, as previously reported, with minor modifications (13). RNA was purified by phenol and chloroform extraction.

cDNA synthesis. cDNA was synthesized with random hexamer primers and Superscript III reverse transcriptase
(Invitrogen, Carlsbad, CA, USA), according to the manufacturer's instructions.

Real-time quantitative RT-PCR. Real-time quantitative RT-PCR analysis was performed with the SYBR Green PCR Master Mix using an Applied Biosystems 7500 Real-Time PCR System according to the manufacturer's instructions (Applied Biosystems, Inc., Foster City, CA, USA). Primers and probes for CD133, CD44 and $\beta$-actin were designed with Primer3 software (Biology Workbench version 3.2; San Diego Supercomputer Center, University of California, San Diego, CA, USA). Sequences were as follows: CD133 specific primers sense, GCTTTGCAATCTCCCTGTTG; antisense, TTGATCCGGGTTCTTACCTG; CD44 specific primers sense, CGGACACCATGGACAAGTTT; antisense, CACGTGGAATACACCTGCAA; and $\beta$-actin sense, ACAGAGCCTCGCCTTTGC; antisense, GCGGCGATA TCATCATCC. PCR was performed in a final volume of $25 \mu \mathrm{l}$ with a SYBR Green PCR Master Mix using $1 \mu \mathrm{l}$ cDNA, $400 \mathrm{nM}$ of each primer for the respective genes. Cycling conditions were $50^{\circ} \mathrm{C}$ for $2 \mathrm{~min}$ and $95^{\circ} \mathrm{C}$ for $10 \mathrm{~min}$, followed by 40 cycles at $95^{\circ} \mathrm{C}$ for $15 \mathrm{sec}$ and $60^{\circ} \mathrm{C}$ for $1 \mathrm{~min}$.

Relative mRNA levels of target genes. Relative mRNA levels were determined by the standard curve method. The standard curves and line equations were generated using 5-fold serially diluted solutions of cDNA from the LoVo colon cancer cell line. All standard curves were linear in the analyzed range with an acceptable correlation coefficient $\left(\mathrm{R}^{2}\right)$. The levels of target gene expression were calculated from the standard curve. Quantitative normalization of cDNA in each sample was performed using the expression of the $\beta$-actin gene as an internal control. Finally, mRNA levels of the target gene were expressed as ratios to $\beta$-actin mRNA levels. Real-time PCR assays were carried out in duplicate for each sample and mean values were used for the calculation of the mRNA levels.

Cell culture. The HT29 human colon cancer cell line was grown in monolayer cultures in RPMI-1640 (Sigma-Aldrich, Inc., St. Louis, MO, USA) supplemented with fetal bovine serum [FBS; 10\% (v/v); Gibco Brl., Tokyo, Japan), glutamine $(2 \mathrm{mM})$, penicillin $(100,000 \mathrm{U} / \mathrm{l})$, streptomycin $(100 \mathrm{mg} / \mathrm{l})$ and gentamycin $(40 \mathrm{mg} / \mathrm{l})$ at $37^{\circ} \mathrm{C}$ in a $5 \% \mathrm{CO}_{2}$ environment.

For routine passage, cultures were spilt 1:10 when they reached $90 \%$ confluence, generally every 3 days. Cells at the fifth to ninth passage were used for all experiments, which were performed with exponentially growing cells.

Radiation exposure. HT-29 cells were plated at a density of $10^{4}$ cells/well in three $100-\mathrm{mm}$ culture dishes (BD Falcon). Exponentially growing cells were plated on a $10-\mathrm{cm}$ dish and irradiated at doses of 1, 2.5 and 5 Gy (CAX-150-20; Chubu Medical Co., Ltd.). Non-irradiated controls were handled identically to the irradiated cells with the exception of the radiation exposure. After irradiation, cultures were kept at $37^{\circ} \mathrm{C}$ and $5 \%$ $\mathrm{CO}_{2}$ in an incubator.

Clonogenic survival assay after radiation. Colony formation assays were performed in triplicates. After 10-14 days of incubation, cells were fixed for $15 \mathrm{~min}$ with $2 \%$ formaldehyde 
and stained for 15 min with $0.5 \%$ crystal violet. Colonies were counted when they contained $>50$ cells. The number of colonies was counted, then the surviving fraction was normalized to the surviving fraction of the corresponding control.

Western blot analysis. Plates were washed in ice-cold PBS, and cold lysis buffer (Tris-buffered saline, $\mathrm{pH} 7.5$, containing $1 \%$ Triton X-100) was added directly to the plates. Cells were scraped from plates, collected and homogenized using a Mixer Mill MM 300 homogenizer (Qiagen Inc., Chatsworth, CA, USA). Supernatants were collected and frozen at $-20^{\circ} \mathrm{C}$ until use. The protein concentration was measured by the BCA protein assay (Pierce, Rockford, IL, USA). Lysate proteins $(20 \mu \mathrm{g})$ were mixed with an equal volume of $2 \mathrm{X}$ Laemmli's loading buffer containing $2 \mathrm{ME}$ and heated at $100^{\circ} \mathrm{C}$ for $5 \mathrm{~min}$. Samples were electrophoretically separated on $12.5 \%$ gradient polyacrylamide gels containing $0.1 \%$ SDS, followed by semi-dry transfer to an Immun-Blot PVDF membrane (Bio-Rad Laboratories, Hercules, CA, USA). The membranes were blocked using 5\% skimmed milk in Tris-buffered saline, pH 7.5, supplemented with $0.1 \%$ Tween-20 (TBS-T). The blots were then incubated with rabbit monoclonal anti-CD133 antibody (Cell Signaling Technology, Inc., Boston, MA, USA) at a 1:1,000 dilution, mouse monoclonal anti-CD44 antibody (R\&D Systems, Inc., Minneapolis, MN, USA) at a 1:1,000 dilution, and mouse monoclonal anti-actin (clone $\mathrm{C} 4$ ) antibody (MP Biomedicals, LLC, Solon, OH, USA) at a 1:400 dilution in $5 \%$ skimmed milk in TBS-T overnight at $4^{\circ} \mathrm{C}$. After washing three times in TBS-T, the blots were incubated with alkaline-phosphatase-conjugated goat anti-rabbit or mouse IgG (Promega, Madison, WI, USA) at a 1:200 dilution in $5 \%$ skimmed milk in TBS-T. Following treatment with an enhanced chemiluminescence detection solution, chemiluminescent signals were visualized using CS Analyzer and AE-6962 Light Capture (ATTO Corp., Tokyo, Japan).

Immunohistochemistry. Sections of FFPE specimens $(2-\mu \mathrm{m})$ were constructed. After deparaffinization and dehydration for antigen unmasking, specimens were brought to a boil in $10 \mathrm{mM}$ sodium citrate buffer. Specimens were then blocked and incubated with primary antibody overnight at $4^{\circ} \mathrm{C}$. The antibody was detected by Envision reagents (Envision kit/ HRP; Dako Cytomation, Denmark). All sections were counterstained with hematoxylin. Primary CD133 rabbit monoclonal antibody and CD44 mouse monoclonal antibody were used in dilutions of 1:150 and 1:1,000 with labeled streptavidin biotin method (LASB2 kit/HRP; Dako Cytomation) added. Negative controls were run simultaneously with pre-immune immunoglobulin.

Statistical analysis. All statistical analyses were carried out using JMP version 5 (SAS Institute Inc., Cary, NC, USA). The value of each target gene was expressed as a median value (inter-quartile range). Associations between gene expression levels (continuous variables) and clinicopathological variables (categorical variables) were evaluated using Mann-Whitney U-test for two groups, or Kruskal-Wallis test for multiple groups. Disease-free survival was calculated from the date of surgery to the date of disease recurrence. Overall survival was calculated from the date of surgery to the date of death due to rectal cancer or last follow-up. Survival was evaluated using the Kaplan-Meier method. The log-rank test was used to compare the cumulative survival durations in the patient groups. A non-parametric receiver operating characteristic (ROC) analysis was performed to calculate the best cutoff values predictive of distant recurrence using Medcalc 7.2 for Windows (Mariakerke, Belgium). P-values of $<0.05$ were considered statistically significant.

\section{Results}

Patient and tumor characteristics. Fifty-two patients were included in this study. The median age was 65 years (range 37-78). The male-to-female ratio was $42: 10$. The post-CRT pathological T stages were pT1-2 (35\%) and pT3-4 (65\%), respectively. Seventeen patients $(33 \%)$ had pathological lymph node metastases. Lymphatic invasion was present in 39 of 52 patients $(75 \%)$, and vascular invasion was present in 30 patients $(58 \%)$. Forty-five tumors $(87 \%)$ showed well- or moderately differentiated adenocarcinoma histology. None of the patients had local recurrence. Patterns of distant recurrence included liver and lung metastases in 3 patients, lung metastases alone in 4 patients, and peritoneal metastasis in 1 patient.

Pathological response to chemoradiotherapy. TRG was as follows: TRG0, 0 patients $(0 \%)$; TRG1, 10 patients $(20 \%)$; TRG2, 22 patients (42\%); TRG3, 20 patients (38\%) and TRG4, 0 patients $(0 \%)$. No TRG4 (complete regression of the primary tumor) was noted in this study because of the unavailability of residual cancer cells.

Associations of post-CRT CD133 and CD44 with clinicopathological variables. The expression values (relative mRNA levels) of CD133 and CD44 were expressed as ratios between the gene of interest (CD133 and CD44) and the internal reference gene $(\beta$-actin), providing a normalization factor for the amount of RNA. Median value of post-CRT CD133 and CD44 mRNA levels was 0.104 (inter-quartile range 0.031-0.422) and 0.442 (inter-quartile range $0.242-0.875$ ), respectively. Associations of post-CRT CD133 and CD44 with clinicopathological variables are summarized in Table I. CD133 was significantly higher in patients with vascular invasion compared to those without $(\mathrm{P}=0.02)$. $\mathrm{CD} 44$ was significantly higher in female patients compared to male patients $(\mathrm{P}=0.02)$. Pathological T1-2 tumors had higher CD44 expression than pathological T3-4 tumors $(\mathrm{P}<0.05)$. As shown in Table II, patients who developed metachronous distant recurrence $(n=8)$ had significantly higher post-CRT CD133 levels compared to the patients without recurrence $(n=44 ; P=0.0209)$. No significant association between distant recurrence and CD44 was observed.

Predictive values of post-CRT CD133 and CD44 for distant recurrence and overall survival. On the basis of these results, ROC analyses were used to identify cutoff values of CD133 or CD44 predictive of distant recurrence and overall survival. A non-parametric ROC analysis showed that the best cutoff values of CD133 and CD44 for distant recurrence were 1.225 
Table I. Association of post-CRT CD133 and CD44 with clinicopathological variables.

\begin{tabular}{|c|c|c|c|c|c|}
\hline Variable & No. & CD133 & P-value & $\mathrm{CD} 44$ & P-value \\
\hline \multicolumn{6}{|l|}{ Gender } \\
\hline Male & 42 & 0.099 & & 0.411 & \\
\hline Female & 10 & 0.132 & 0.83 & 1.063 & 0.02 \\
\hline \multicolumn{6}{|c|}{ Age (years; median 65) } \\
\hline$\leq 65$ & 26 & 0.065 & & 0.457 & \\
\hline$>65$ & 26 & 0.114 & 0.29 & 0.408 & 0.35 \\
\hline \multicolumn{6}{|c|}{ Pathological T category } \\
\hline T1-2 & 18 & 0.087 & & 0.734 & \\
\hline T3-4 & 34 & 0.125 & 0.30 & 0.356 & $<0.05$ \\
\hline \multicolumn{6}{|c|}{ Pathological N category } \\
\hline Present & 17 & 0.097 & & 0.350 & \\
\hline Absent & 35 & 0.107 & 0.56 & 0.524 & 0.08 \\
\hline \multicolumn{6}{|l|}{ Histology } \\
\hline Well/moderate & 45 & 0.107 & & 0.452 & \\
\hline Poor-mucinous & 7 & 0.097 & 0.89 & 0.350 & 0.95 \\
\hline \multicolumn{6}{|c|}{ Lymphatic invasion } \\
\hline Present & 39 & 0.148 & & 0.429 & \\
\hline Absent & 13 & 0.032 & 0.06 & 0.773 & 0.41 \\
\hline \multicolumn{6}{|l|}{ Vascular invasion } \\
\hline Present & 30 & 0.209 & & 0.429 & \\
\hline Absent & 22 & 0.047 & 0.02 & 0.468 & 0.32 \\
\hline \multicolumn{6}{|c|}{ Tumor Regression Grading } \\
\hline TRG1 & 10 & 0.123 & & 0.617 & \\
\hline TRG2 & 22 & 0.099 & & 0.356 & \\
\hline TRG3 & 30 & 0.079 & 0.78 & 0.582 & 0.21 \\
\hline
\end{tabular}

$\mathrm{T}$ and $\mathrm{N}$ categories indicate extent of the primary tumor and presence of lymph node metastasis. Values for each target gene are expressed as median values. Bold type indicates a significant value.

Table II. Association of post-CRT CD133 and CD44 with distant recurrence.

\begin{tabular}{|c|c|c|c|}
\hline Target genes & Patients with distant recurrence $(n=8)$ & Patients without distant recurrence $(n=44)$ & P-value \\
\hline CD133 & $0.248(0.026-1.412)$ & $0.104(0.032-0.291)$ & 0.0209 \\
\hline CD44 & $0.543(0.285-0.627)$ & $0.430(0.228-1.118)$ & 0.4599 \\
\hline
\end{tabular}

Value for each target gene is expressed as the median value (inter-quartile range).

and 0.64 , respectively. The best cutoff values of CD133 and CD44 for overall survival were 0.21 and 1.648 , respectively. As shown in Fig. 1, patients with post-CRT CD133 above the cutoff value (High) showed significantly worse disease-free survival (log-rank test; $\mathrm{P}=0.0229$ ).

CD133 and CD44 expression in paired pre-and post-CRT specimens. To confirm whether CD133 and CD44 expression was altered during CRT, expression of each gene was compared between matched pre-CRT and post-CRT specimens $(n=30)$. As shown in Fig. 2, CD133 was significantly increased in post-CRT specimens $(\mathrm{P}=0.01)$, compared to pre-CRT biopsy specimens. By contrast, CD44 was signifi- cantly decreased in residual cancer cells of the post-CRT specimens $(\mathrm{P}<0.001)$.

Clonogenic survival of HT-29 cells after irradiation. Significant reduction in clonogenic survival was observed in the radiation-treated HT-29 cells (Fig. 3A). Survival fractions of approximately 23, 7 and 5\% were noted when cells were irradiated with 1,2.5 and $5 \mathrm{~Gy}$, respectively.

Post-irradiation CD133 and CD44 expression in clonogenic surviving cells. As shown in Fig. 3B and C, a single dose of 1, 2.5 and 5 Gy radiation increased CD133 protein levels, compared to controls as determined by western blot analysis. 

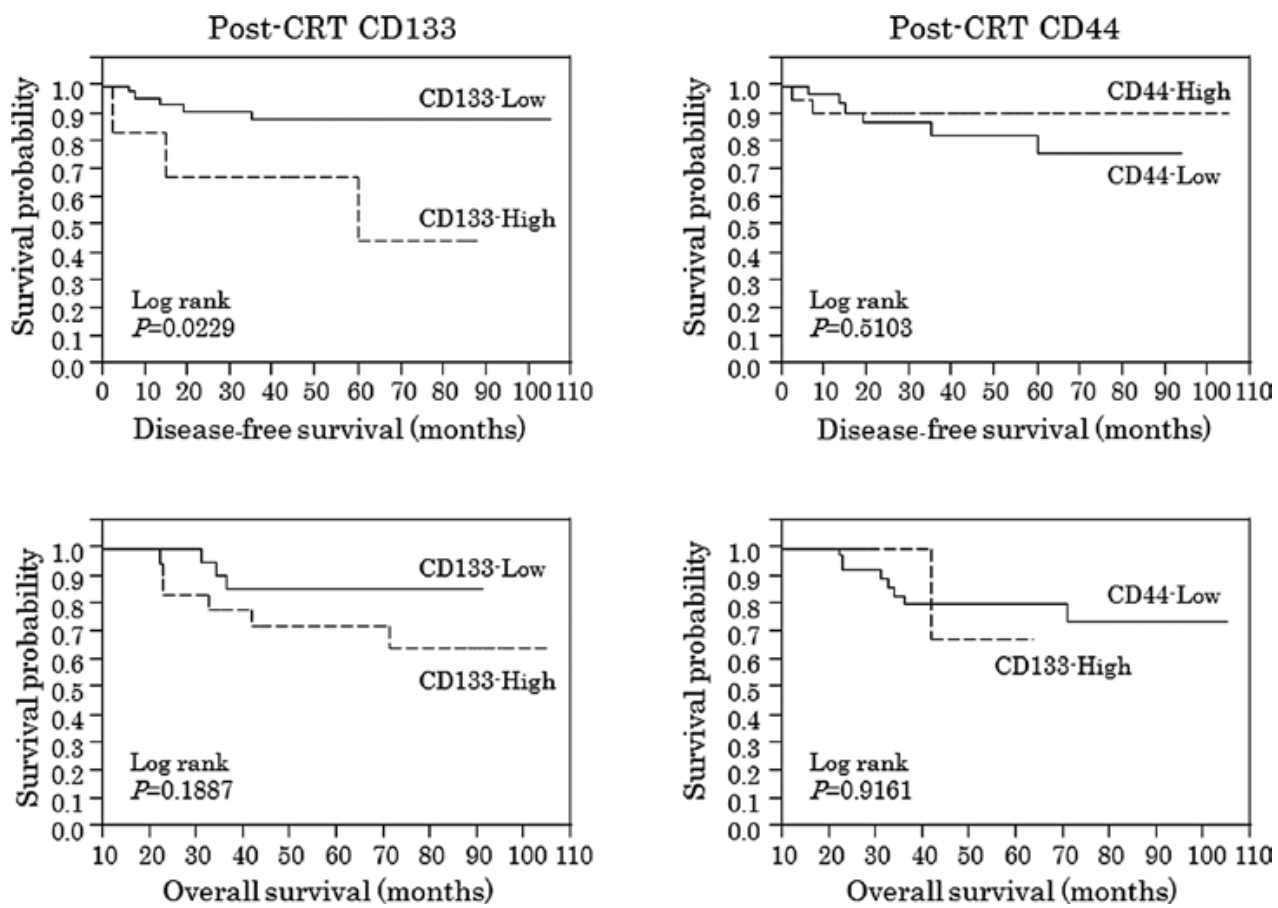

Figure 1. Survival according to post-CRT CD133 or CD44 expression. Patients with post-CRT CD133 above the cutoff value (High) showed significantly worse disease-free survival (log-rank test; $\mathrm{P}=0.0229)$.

$\mathrm{CD} 133$

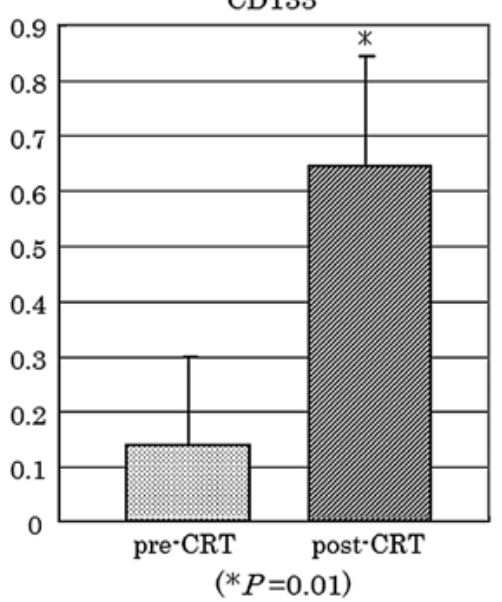

CD44

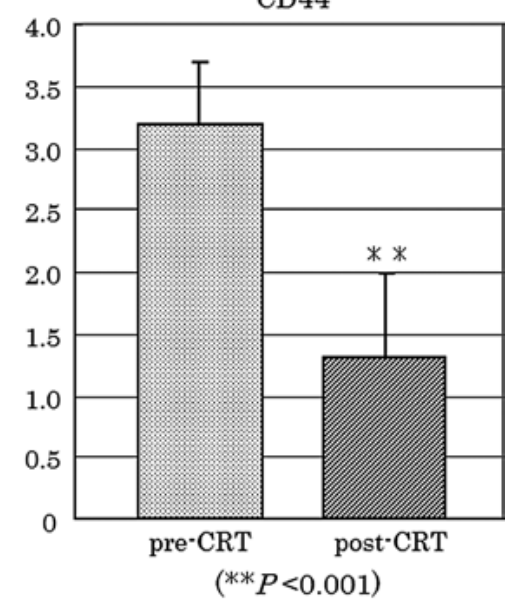

Figure 2. CD133 and CD44 expression in paired pre- and post-CRT specimens. CD133 was higher in post-CRT specimens than that in the pre-CRT specimens $(\mathrm{P}=0.01)$. By contrast, $\mathrm{CD} 44$ was lower in the post-CRT residual cancer cells than in the pre-CRT biopsy specimens $(\mathrm{P}<0.001)$. Data are presented as the means \pm standard error

Densitometric analysis showed that CD133 was 1.4 times increased at 5 Gy radiation with respect to the control. By contrast, radiation decreased CD44 protein levels, regardless of the radiation dose.

Immunohistochemistry for CD133 and CD44 in residual cancer cells. The minority of residual cancer cells had immunoreactive CD133 and CD44 expression within the entire residual cancer. CD133 was observed diffusely in the cytoplasm of residual cancer cells, and was also located at an apical/endoluminal surface (Fig. 4A and C). Immunoreactive CD44 expression was found as membranous staining (Fig. 4B and D). There was no obvious concordance between CD133 and CD44 positivity of the residual cancer cells.

\section{Discussion}

The presence of CSCs in the primary tumor appears to have prognostic significance in several types of malignancies $(14,15)$. In primary colorectal cancer, CD133 expression has been also reported to be a significant prognostic marker $(16,17)$. In this study, post-CRT CD133 in residual cancer cells was significantly associated with metachronous distant recurrence and poor disease-free survival of rectal cancer patients, although pre-CRT CD133 in the primary tumor did not correlate with clinical outcome (data not shown). There are several potential explanations of these results. First, the small sample size of pre-CRT biopsies $(n=30)$ may have affected the results. Second, CRT-resistant surviving cells, which are residual 
A

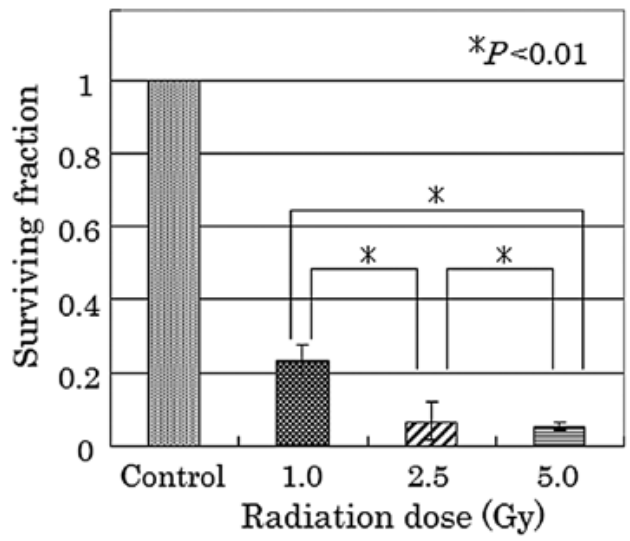

C

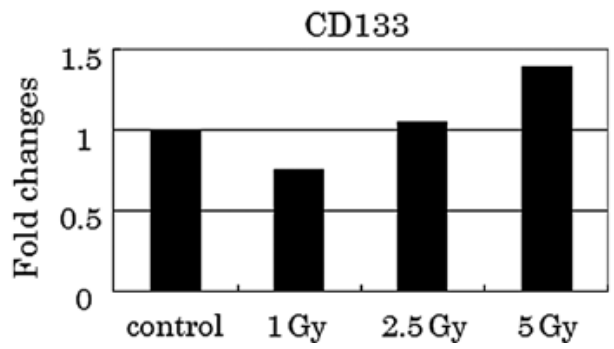

B

actin

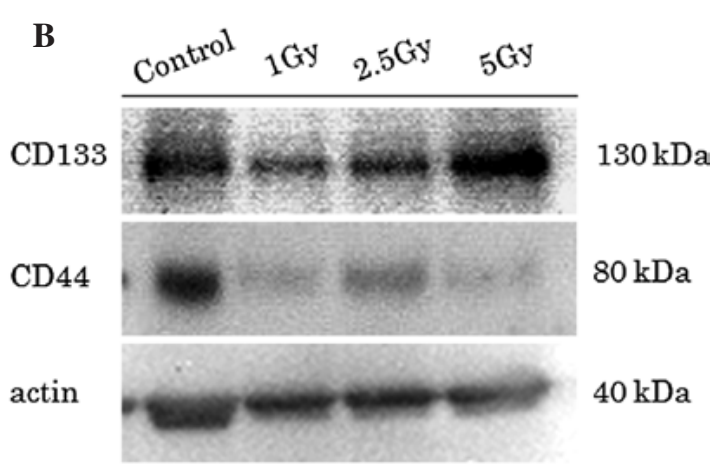

CD44

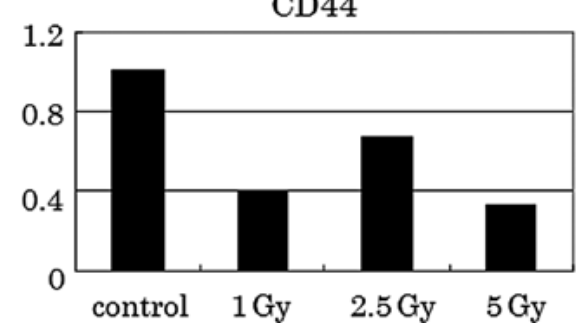

Figure 3. (A) Clonogenic survival assay of radiation-treated HT-29 cells. Clonogenic survival fractions of HT-29 cells were decreased in a radiation dose-dependent manner. Data are presented as the means \pm standard deviation. Asterisk indicates statistically significant difference. (B and C) Expression of CD133 and CD44 in radiation-treated HT-29 cells. CD133 protein was increased in a radiation dose-dependent manner. By contrast, CD44 protein was decreased after irradiation.
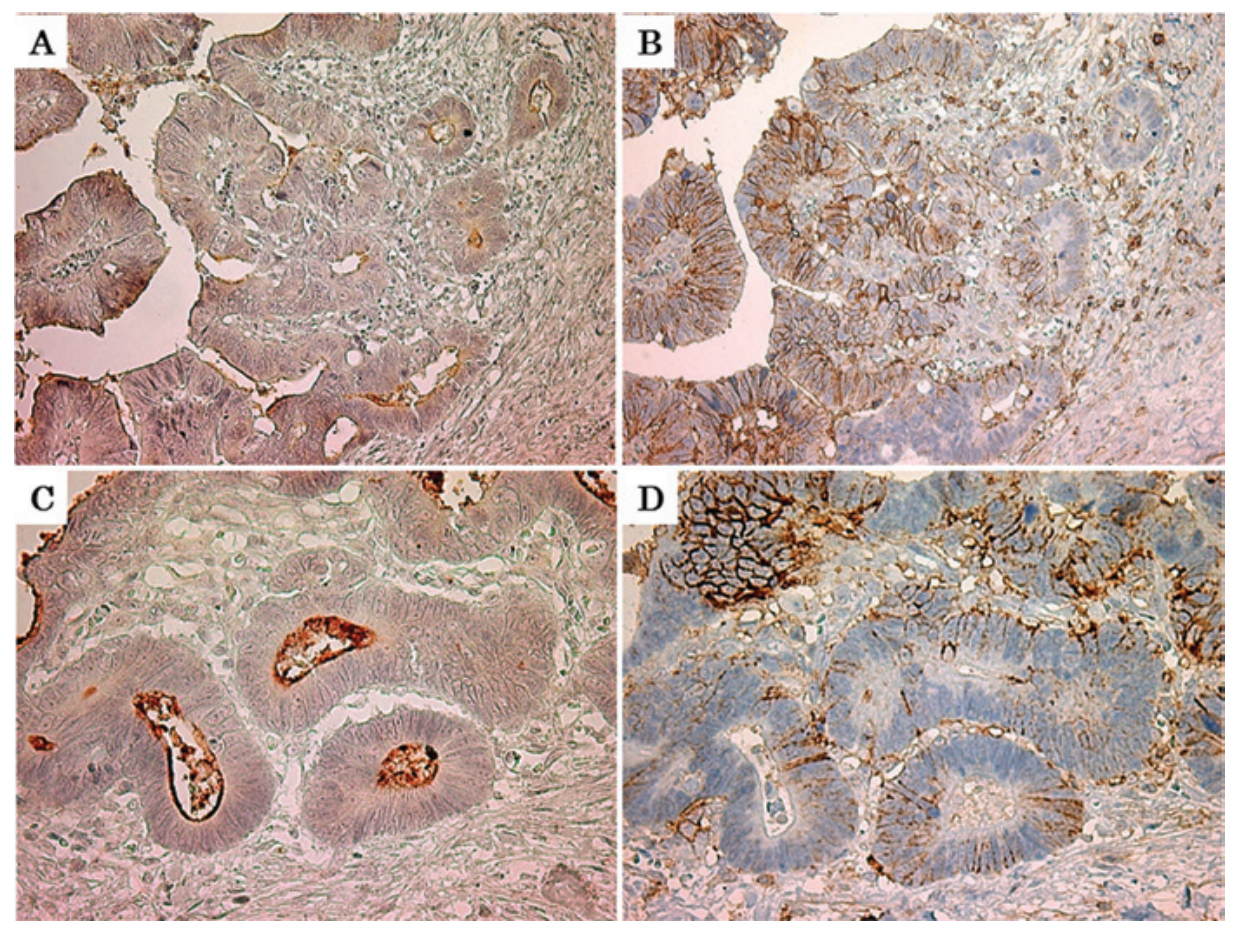

Figure 4. Immunoreactive CD133 and CD44 expression in residual cancer cells. Immunoreactive CD133 protein was located in the cytoplasm and at an apical/ endoluminal surface of residual cancer cells (A: magnification, x200; C: magnification, x400). Immunoreactive CD44 protein was located at the surface membrane of residual cancer cells (B: magnification, x200; D: magnification, x400). No obvious concordance was found between CD133 and CD44 positivity of residual cancer cells.

cancer cells in post-CRT specimens, may have much more putative CSCs than the pre-CRT primary tumor, so that postCRT CD133 expression preferentially correlates with clinical outcome. Third, CRT may alter CD133 expression, resulting in a difference in the CD133 expression status between pre-CRT and post-CRT specimens.

To clarify whether irradiation may change CD133 expression, we compared CD133 expression in radiation-treated 
HT-29 cells to that in non-irradiated cells. CD133 protein levels were increased in a radiation dose-dependent manner, despite the decreased number of clonogenic radiation-surviving HT-29 cells. These in vitro findings were consistent with the fact that CD133 mRNA levels were increased after CRT in comparison to levels before CRT. These results suggest that not only residual cancer cells surviving CRT may enrich the population of putative CSCs expressing CD133, but also CRT may induce CD133 expression. CD44 has been also considered as an important marker for isolating colon CSCs (7-9). However, there was no significant association between post-CRT CD44 and clinical outcome. Furthermore, no significant correlation between CD133 and CD44 mRNA levels was observed in preand post-CRT cancer cells (data not shown).

Immunohistochemically, there was no clear concordance between CD133 and CD44 positivity of residual cancer cells. This may support the hypothesis that immunoreactive CD133-positive and CD44-positive cells do not colocalize in colorectal cancer specimens (8).

Introduction of pre-operative CRT and TME for rectal cancer has improved the sphincter preservation rate, local pelvic control and survival (18). However, metachronous distant recurrence remains the major cause of mortality in rectal cancer patients treated with CRT followed by TME (19). Our data indicate that CD133, but not CD44, in residual cancer cells may be significantly relevant to the clinical outcome of rectal cancer patients who are treated with pre-operative CRT followed by TME. However, data from this study should be interpreted with caution. The main limitation of this study was the relatively small number of patients $(n=52)$, including only 8 patients with distant recurrence. Large prospective trials are required to confirm the validity of the predictive value of postCRT CD133 for distant recurrence and survival.

In conclusion, CD133, but not CD44, was increased in the radiation-resistant surviving colon cancer cells. Post-CRT CD133 in residual cancer cells may predict metachronous distant recurrence and poor survival of rectal cancer patients after CRT.

\section{References}

1. Eyler CE and Rich JN: Survival of the fittest: cancer stem cells in therapeutic resistance and angiogenesis. J Clin Oncol 26 2839-2845, 2008.

2. Baumann M, Krause M and Hill R: Exploring the role of cancer stem cells in radioresistance. Nat Rev Cancer 8: 545-554, 2008.
3. Bao S, Wu Q, McLendon RE, et al: Glioma stem cells promote radioresistance by preferential activation of the DNA damage response. Nature 444: 756-760, 2006.

4. Tsuchida R, Das B, Yeger H, et al: Cisplatin treatment increases survival and expansion of a highly tumorigenic side-population fraction by upregulating VEGF/Flt1 autocrine signaling. Oncogene 27: 3923-3934, 2006.

5. Ricci-Vitiani L, Lombardi DG, Pilozzi E, Biffoni M, Todaro M, Peschle $\mathrm{C}$ and de Maria R: Identification and expansion of human colon-cancer-initiating cells. Nature 445: 111-115, 2007.

6. O'Brien CA, Pollett A, Gallinger S and Dick JE: A human colon cancer cell capable of initiating tumour growth in immunodeficient mice. Nature 445: 106-110, 2007.

7. Haraguchi N, Ohkuma M, Sakashita H, et al: CD133 ${ }^{+} \mathrm{CD} 44^{+}$ population efficiently enriches colon cancer initiating cells. Ann Surg Oncol 15: 2927-2933, 2008.

8. Du L, Wang H, He L, et al: CD44 is of functional importance for colorectal cancer stem cells. Clin Cancer Res 14: 6751-6760, 2008.

9. Chu P, Clanton DJ, Snipas TS, et al: Characterization of a subpopulation of colon cancer cells with stem cell-like properties. Int J Cancer 124: 1312-1321, 2009.

10. Yoshikawa R, Kusunoki M, Yanagi H, et al: Dual antitumor effects of 5-fluorouracil on the cell cycle in colorectal carcinoma cells: a novel target mechanism concept for pharmacokinetic modulating chemotherapy. Cancer Res 61: 1029-1037, 2001.

11. Sobin LH and Fleming ID: TNM Classification of Malignant Tumors, fifth edition (1997). Union Internationale Contre le Cancer and the American Joint Committee on Cancer. Cancer 80: 1803-1804, 1997.

12. Dworak O, Keilholz L and Hoffmann A: Pathological features of rectal cancer after preoperative radiochemotherapy. Int J Colorectal Dis 12: 19-23, 1997.

13. Bijwaard KE, Aguilera NS, Monczak Y, Trudel M, Taubenberger JK and Lichy JH: Quantitative real-time reverse transcription-PCR assay for cyclin D1 expression: utility in the diagnosis of mantle cell lymphoma. Clin Chem 47: 195-201, 2001.

14. Liu R, Wang X, Chen GY, et al: The prognostic role of a gene signature from tumorigenic breast-cancer cells. N Engl J Med 356: 217-226, 2007.

15. Zeppernick F, Ahmadi R, Campos B, et al: Stem cell marker CD133 affects clinical outcome in glioma patients. Clin Cancer Res 14: 123-129, 2008

16. Horst D, Kriegl L, Engel J, Kirchner T and Jung A: CD133 expression is an independent prognostic marker for low survival in colorectal cancer. Br J Cancer 99: 1285-1289, 2008.

17. Kojima M, Ishii G, Atsumi N, Fujii S, Saito N and Ochiai A: Immunohistochemical detection of CD133 expression in colorectal cancer: a clinicopathological study. Cancer Sci 99: 1578-1583, 2008.

18. Bosset JF, Collette L, Calais G, et al; EORTC Radiotherapy Group Trial 22921: Chemotherapy with preoperative radiotherapy in rectal cancer. N Engl J Med 355: 1114-1123, 2007.

19. Bujko K, Michalski W, Kepka L, et al; Polish Colorectal Study Group: Association between pathologic response in metastatic lymph nodes after preoperative chemoradiotherapy and risk of distant metastases in rectal cancer: an analysis of outcomes in a randomized trial. Int J Radiat Oncol Biol Phys 67: 369-377, 2007. 